\title{
DFT study of graphene doping due to metal contacts
}

\author{
P. Khakbaz*, F. Driussi*, A. Gambi*, \\ P. Giannozzi ${ }^{\dagger}$, S. Venica* ${ }^{*}$ D. Esseni* \\ *DPIA, University of Udine, Udine, Italy \\ ${ }^{\dagger}$ DMIF, University of Udine, Udine, Italy \\ Email: francesco.driussi@uniud.it
}

\author{
A. Gahoi ${ }^{\ddagger}$, S. Kataria ${ }^{\ddagger}$, M.C. Lemme ${ }^{\ddagger}$ \\ ${ }^{\ddagger}$ RWTH Aachen University, Aachen, Germany \\ $\S$ AMO GmbH, Advanced Microelectronic Center, Aachen, Germany
}

\begin{abstract}
The experimental results of Metal-graphene (M-G) contact resistance $\left(R_{C}\right)$ have been investigated in-depth by means of Density Functional Theory (DFT). The simulations allowed us to build a consistent picture explaining the $R_{C}$ dependence on the metal contact materials employed in this work and on the applied back-gate voltage. In this respect, the M-G distance is paramount in determining the $R_{C}$ behavior.
\end{abstract}

Index Terms-Graphene, Contacts, DFT

\section{INTRODUCTION}

In recent years, graphene raised great interest for many electronic applications [1], [2]. However, metal-graphene (MG) contacts still severely degrade the electrical performance of graphene based devices because of the large contact resistance $\left(R_{C}\right)$. For instance, $R_{C}$ largely degrades the output conductance and the maximum oscillation frequency of grapheneFETs (GFETs) [1], [3]. Thus, to boost the graphene technology, design strategies to optimize $R_{C}$ are urgently required to make graphene a viable solution for high performance electronic devices [4].

In this respect, for a proper contact engineering, the physics underlying the conduction through $\mathrm{M}-\mathrm{G}$ contacts needs to be completely understood. Therefore, in this work we made use of Density Functional Theory (DFT) simulations to interpret the experimental $R_{C}$ values obtained for different metal materials [5] and to gain an insight into the $M-G$ contact physics.

\section{EXPERIMENTAL BEHAVior OF M-G CONTACT RESISTANCE}

Figure 1 reports the contact resistance values measured on back-gated TLM structures with contacts to graphene fabricated with nickel, coppper and gold. Details concerning the measured devices and the exploited characterization technique can be found in [6], [7].

As it can be seen, $R_{C}$ largely depends on the applied backgate voltage $V_{B G}$. Furthermore, despite the similar graphene quality between the different samples (not shown) [8], the $R_{C}$ values do depend on the metal contact, with larger $R_{C}$ values for $\mathrm{Ni}$ and smaller $R_{C}$ values for Au. This indicates that the $\mathrm{M}-\mathrm{G}$ interactions influence the electrical properties of the $\mathrm{M}-$ G contact, which is not a surprising result [9].

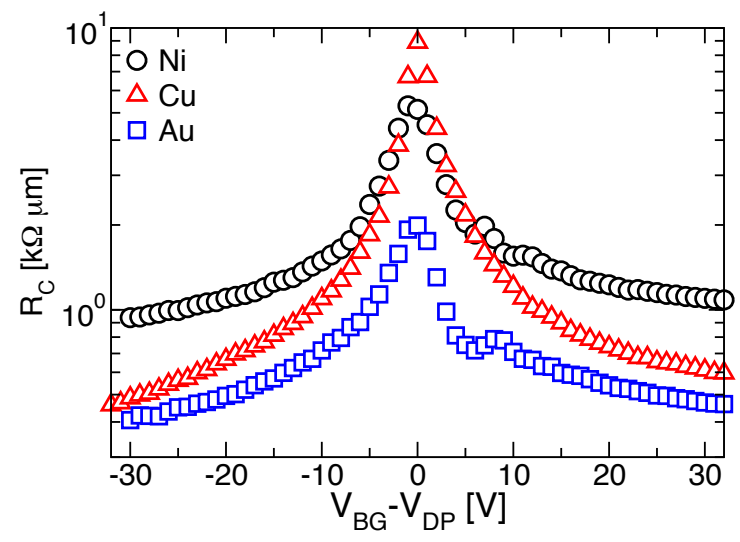

Fig. 1: Experimental contact resistance $R_{C}$ as a function of $V_{B G}$ for samples with $\mathrm{Ni}, \mathrm{Cu}$ and Au contacts. $V_{D P}$ is the $V_{B G}$ corresponding to the Dirac Point, at which the graphene resistance is maximum.

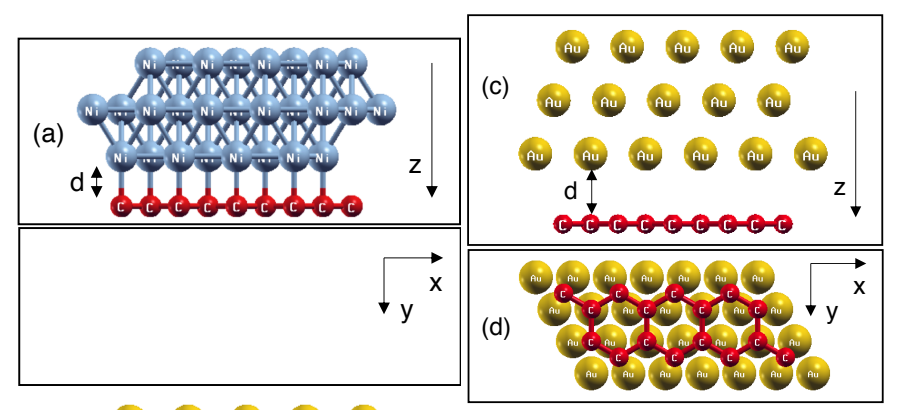

Fig. 2: Lateral (a) and bottom (b) view of the simulated Ni-G structure. A $1 \times 1$ graphene cell with a lattice constant of $a=0.246 \mathrm{~nm}$ is considered. For the $\mathrm{Cu}-\mathrm{G}$ stack, we used the same cell. Lateral (c) and bottom (d) view of the simulated $\mathrm{Au}-\mathrm{G}$ structure. The Au lattice is matched with a $2 \times 2$ graphene supercell [10].

\section{DENSITY FUnCTIONAL TheORY SIMUlations}

To interpret the experimental results in Fig. 1, we performed DFT simulations with the Quantum ESPRESSO suite [11]. We built 3-layer-thick clusters for $\mathrm{Ni}, \mathrm{Cu}$ and $\mathrm{Au}$ contacts and we matched the 111 metal surface with the graphene $(\mathrm{G})$ lattice, which primitive cell as an in-plane lattice constant of $a=0.246 \mathrm{~nm}$ [10] (Fig. 2). We verified that the three metal layers are sufficient to develop a band structure consistent with the bulk metallic material (not shown). In the supercell, we 
also included a vacuum region of about $2 \mathrm{~nm}$ and we applied dipole correction in order to avoid spurious interactions between periodic images of the slab [11].

We used local-spin density approximation, plane-wave basis sets and gradient-corrected exchange-correlation functionals (Perdew-Burke-Ernzerhof) for $\mathrm{Ni}, \mathrm{Cu}, \mathrm{Au}$ and $\mathrm{C}$ atoms. Van der Waals forces employing the Grimme-D2 empirical method were also accounted for. Energy minimisation allowed us to obtain the equilibrium geometry and the minimum energy distance (MED) between graphene and $\mathrm{Au}(d=0.31 \mathrm{~nm}), \mathrm{Cu}$ $(d=0.31 \mathrm{~nm})$ or $\mathrm{Ni}(d=0.21 \mathrm{~nm})$ contacts, in agreement with [10].

Figure 3 reports the energy bands of the $\mathrm{Cu}-\mathrm{G}$ (a) and $\mathrm{Au}-$ G (b) stacks at MED: it is worth to notice that these bands are simply the superimposition of metal and graphene bands (not shown) and no hybridization of the metal and graphene orbitals is expected [10]. Here, the typical Dirac cone of graphene at the $K$ point is clearly visible. Furthermore, opposite spin states are degenerate, because $\mathrm{Cu}, \mathrm{Au}$ and graphene are diamagnetic.

Figure 4 shows instead the energy bands of the $\mathrm{Ni}-\mathrm{G}$ structure at two Ni-G distances: MED $d=0.21 \mathrm{~nm}$ (a) and $d=0.3 \mathrm{~nm}$ (b). Spin degeneracy is here lifted, because $\mathrm{Ni}$ is ferromagnetic. The Ni-G interaction is very strong and the hybridization of graphene and $\mathrm{Ni}$ orbitals changes the bands compared to isolated materials. Indeed, at the $K$ point, it is
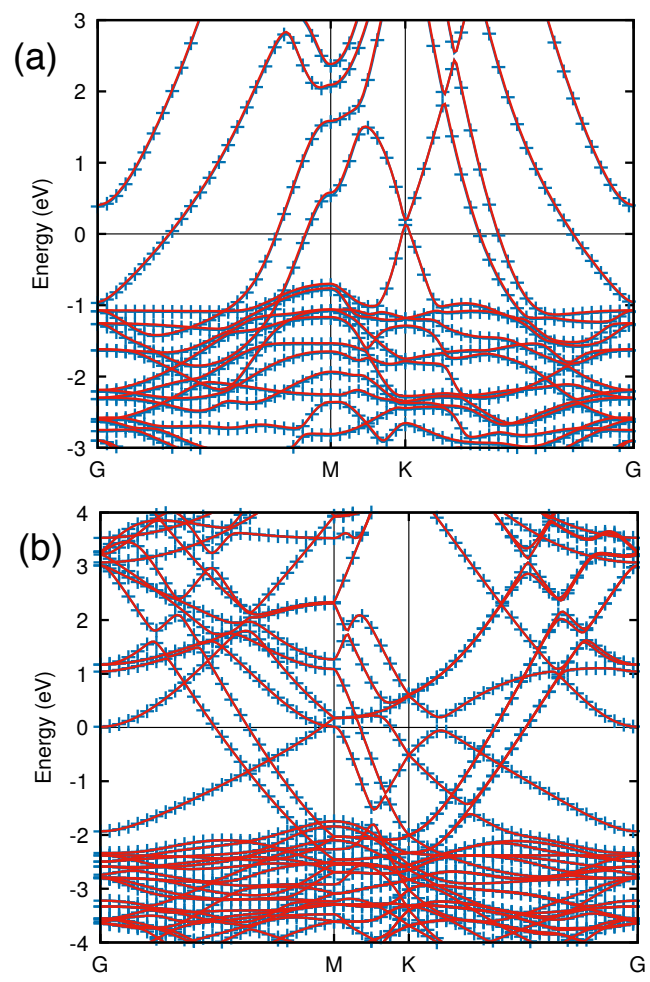

Fig. 3: Energy bands of $\mathrm{Cu}-\mathrm{G}$ (a) and $\mathrm{Au}-\mathrm{G}$ (b) stacks at minimum energy distance $(d=0.31 \mathrm{~nm})$. For both cases bands are the superimposion of metal and graphene bands and the Dirac cone is visible at the $K$ point. Note that the $\mathrm{Au}-\mathrm{G}$ supercell is based on a $2 \times 2$ graphene cell, resulting in the folding of graphene bands. Blue crosses/lines are for spin up, red lines are for spin down.
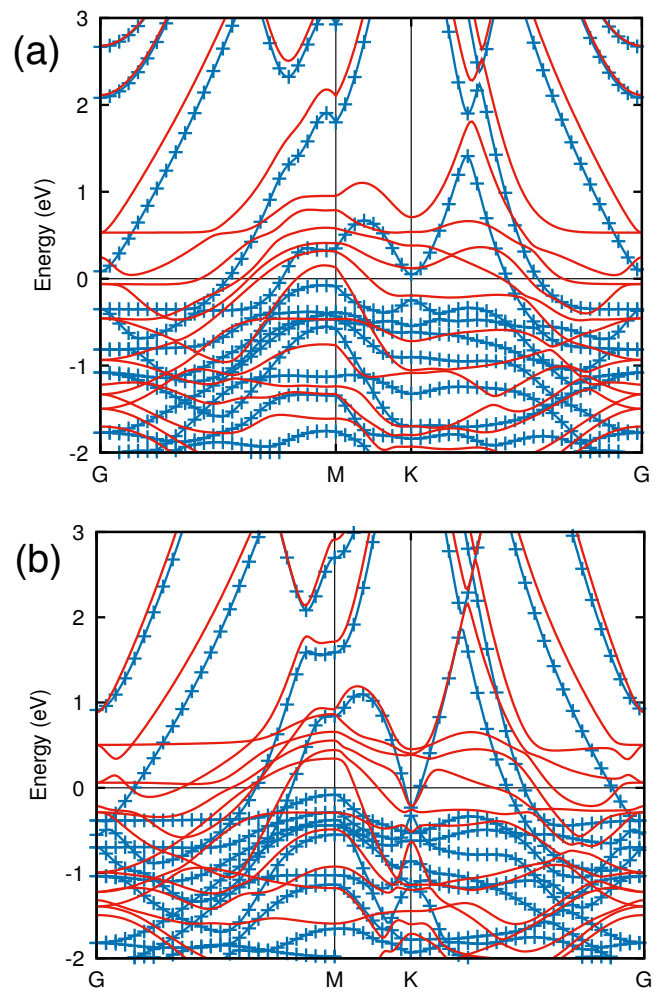

Fig. 4: Energy bands of Ni-G stack for two $d$ values: (a) MED, $d=0.21$ $\mathrm{nm}$; (b) $d=0.3 \mathrm{~nm}$. Ni induces gaps in graphene and the Dirac cone at the $K$ point vanishes. Blue crosses/lines are for spin up, while red lines are for spin down.

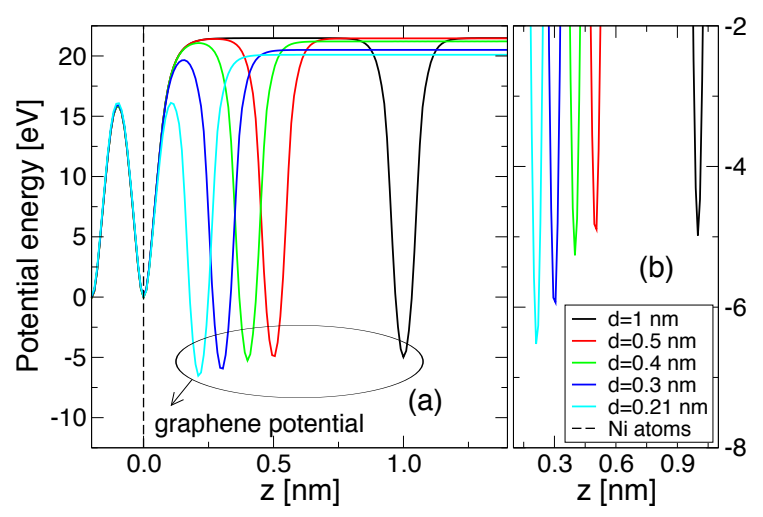

Fig. 5: (a) Potential energy along $z$ (perpendicular to graphene) of the $\mathrm{Ni}-\mathrm{G}$ stack for different $d$ values. The energy reference has been taken at the $\mathrm{Ni}$ atoms position. (b) Zoom in the graphene region. The graphene relative energy with respect to $\mathrm{Ni}$ atoms changes when the layers are approaching, indicating the charging of the two materials.

no longer possible to identify the Dirac cone of graphene for any of the distances in Fig. 4 [10].

\section{Metal-INDUCED Doping OF GRAPHEnE}

Figure 5(a) shows the potential energy calculated along the $z$ direction of the Ni-G stack (perpendicular to graphene, Fig. 2) and averaged over the $x-y$ plane. The energy profiles are reported for different $\mathrm{Ni}-\mathrm{G}$ distances $(d)$ and they describe the variation of the energy of the two materials when they are 


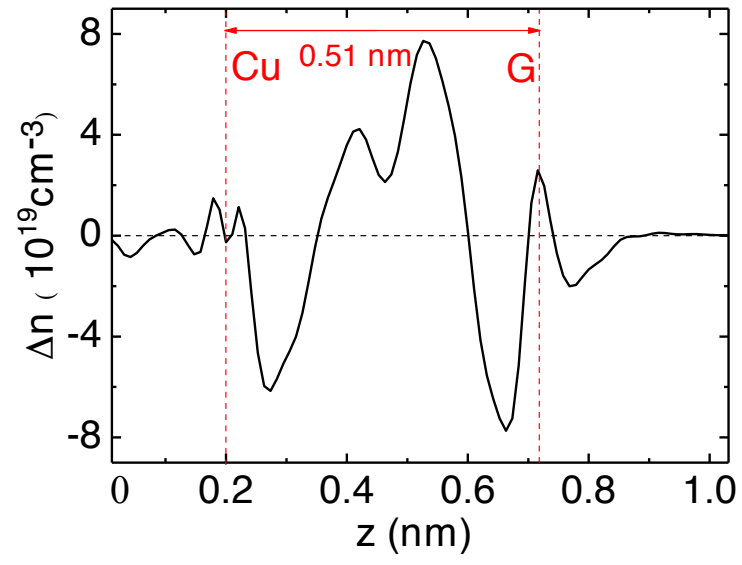

Fig. 6: Induced net charge along $z$ (perpendicular to graphene) for the $\mathrm{Cu}-$ $\mathrm{G}$ stack at $d=0.51 \mathrm{~nm}$. Vertical dashed lines are the positions of graphene and of the closest $\mathrm{Cu}$ layer.

approaching. The lower peaks are located at the atom positions and, by reducing $d$, the graphene peak lowers with respect to the Ni peaks (see Fig. 5(b)), indicating the charging of the two materials.

We studied the charge transfer due to the chemical interaction between metal and graphene by calculating the difference $(\Delta n)$ between the valence electron density of the M-G stacks and of the isolated metal and graphene layers [12], [13]. Fig. 6 shows a typical example of these calculations averaged over the graphene plane for the $\mathrm{Cu}-\mathrm{G}$ stack (at $d=0.51 \mathrm{~nm}$ ). The interaction between the metal and graphene induces a non negligible charge transfer, with the building up of dipoles along the stack $(\Delta n>0$ corresponds to negative charge, $\Delta n<0$ indicates positive charge). In this respect, $R_{C}$ values are expected to be largely affected by the charge in the graphene underneath the contact [10]. Thus, there is the need of estimating the graphene doping due to the metal proximity. However, from this plot it is not possible to evaluate the metalinduced $\mathrm{G}$ doping, since it is not straightforward to distinguish between the charge belonging either to graphene or to the $\mathrm{Cu}$ contact [8].

\section{A. Graphene doping extraction through Bader analysis}

In order to extract a dependable value for the graphene doping, also avoiding spurious contributions by the charge redistribution due to Pauli repulsion [13], we made used of the Bader analysis to calculate the charge variation of each $\mathrm{C}$ atom induced by the presence of the metal contact [14]. Then, we converted this charge into an areal doping density of the graphene sheet.

Figure 7 shows the calculated doping for the different $M-G$ contacts and for several $d$ values down to the MED of each structure. $\mathrm{Cu}$ and $\mathrm{Ni}$ induce $n$-type doping in graphene, while, for the Au contact, graphene becomes $p$-type [13]. It is evident that, at small $d$, the metals largely dope the graphene, with a huge doping value in the case of the Ni-G stack at MED. However, under these circumstances, it becomes difficult to interpret the $\mathrm{Ni}-\mathrm{G}$ stack in terms of a $\mathrm{Ni}$ and a graphene

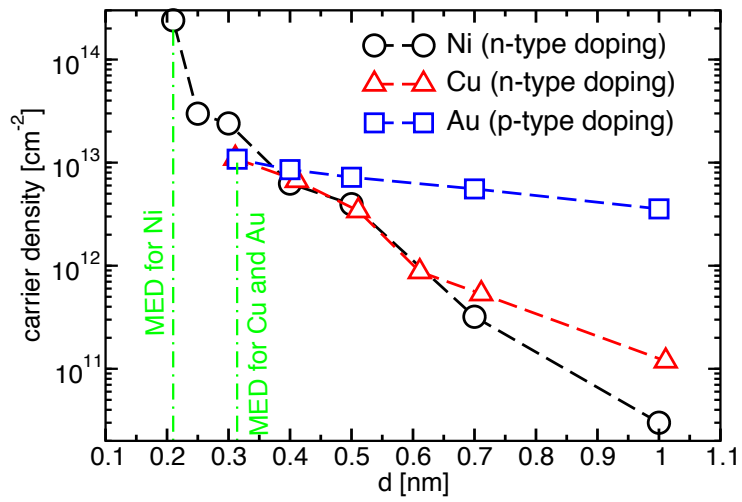

Fig. 7: Graphene doping as a function of the distance $d$, calculated through Bader analysis. Au-G contact shows long range interaction, while $\mathrm{Ni}-\mathrm{G}$ and $\mathrm{Cu}-\mathrm{G}$ stacks show shorter range interactions. Minimum energy distance (MED) values are highlighted for the three $\mathrm{M}-\mathrm{G}$ contacts.
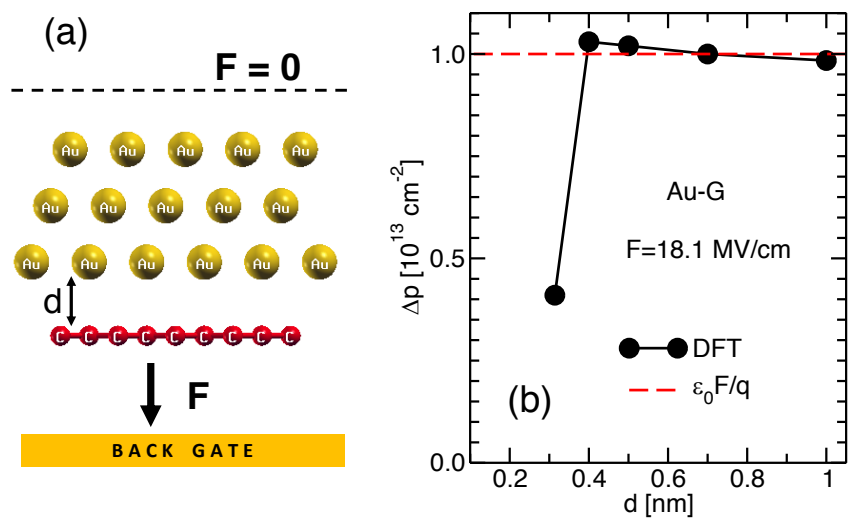

Fig. 8: Back gated Au-G stack: (a) Sketch of the applied electric field $F$ inducing a total charge $\varepsilon_{0} F / q$ in the stack; (b) Graphene hole density variation $\Delta p$ determined via Bader analysis and compared to the total charge $\varepsilon_{0} F / q$.

sub-systems, because of the strong hybridization of the two materials already discussed in Fig. 4.

Figure 7 demonstrates the very different dependence on $d$ of the induced graphene doping for the different metals, with a very long range interaction between $\mathrm{Au}$ and graphene. For $\mathrm{Ni}$ and $\mathrm{Cu}$, instead, the interaction with graphene has a much shorter range.

This trend for distances larger than the MED is consistent with the experimental results of Fig. 1. Indeed, the Au-G stack shows the lowest $R_{C}$ values suggesting a significant doping of graphene, while the $\mathrm{Ni}-\mathrm{G}$ system has the worst $R_{C}$, thus indicating a weak $\mathrm{Ni}-\mathrm{G}$ interaction. The comparison between the experimental $R_{C}$ behavior and the simulations in Fig. 7 suggests that the actual $M-G$ distance in the fabricated contacts is larger than $0.5 \mathrm{~nm}$, thus leading to an inefficient doping of graphene by the $\mathrm{Ni}$ (with the worst $R_{C}$ ). Au instead is still effective in doping the underlying graphene (with the best $R_{C}$ ), even at a non optimal $\mathrm{Au}-\mathrm{G}$ distance. 


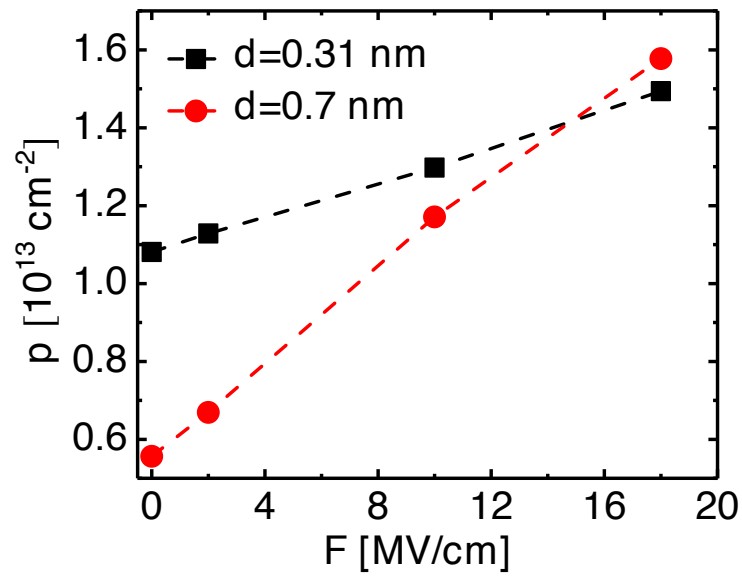

Fig. 9: Back gated Au-G stack: Graphene doping density $p$ determined via Bader analysis at different back-gate electric fields $F$ for two $\mathrm{Au}-\mathrm{G}$ distances. At MED, the gold contact is more effective in defining the doping of the underlying graphene, also reducing the $p$ dependence on $F$.

\section{B. $R_{C}$ dependence on the back-gate voltage}

Finally, in order to interpret the experimentally observed $R_{C}$ dependence on $V_{B G}$, we used DFT simulations and Bader analysis to study also the influence on the graphene doping of a back-gate induced electric field $F$. In particular, as sketched in Fig. 8(a), we repeated the analysis in Fig. 7 on $\mathrm{M}-\mathrm{G}$ stacks undergoing the electrostatic effect of gate plate, inducing $F$ along the $z$ direction perpendicular the graphene sheet The dipole correction option in the Quantum Espresso suite allowed us to apply a non null $F$ only at the graphene side of the $\mathrm{M}-\mathrm{G}$ stack, thus reproducing an electrostatic configuration similar to that in the experiments [8].

Figure $8(\mathrm{~b})$ reports the $F$ induced variation of the graphene doping $(\Delta p)$, calculated as the difference between the charge configuration in the $\mathrm{Au}-\mathrm{G}$ stack subject to the field $F$ and that for the unperturbed stack (with $F=0$, thus without the gate plate). The results show that, for $d \geq 0.4 \mathrm{~nm}$, the $\Delta p$ accounts for the entire charge $\varepsilon_{0} F / q$ induced in the Au-G stack, whereas at the MED $d=0.31 \mathrm{~nm}$ only about the $40 \%$ of $\varepsilon_{0} F / q$ is induced in the graphene.

Figure 9 compares the dependence of the graphene doping $p$ on the applied $F$ value, for Au-G stacks at the MED and at $d=0.7 \mathrm{~nm}$. At MED, the gold contact is more effective in defining the doping of the underlying graphene, also reducing the $p$ dependence on $F$, that is more pronounced for $d=$ $0.7 \mathrm{~nm}$. Therefore, the large $R_{C}$ dependence on the $V_{B G}$ bias in the experiments of Fig. 1 is consistent with a distance $d \geq$ $0.4 \mathrm{~nm}$, such that $V_{B G}$ can still modulate the graphene charge to a large extent.

\section{CONCLUSIONS}

In summary, our simulations show that $\mathrm{Ni}$ and $\mathrm{Cu}$ largely dope graphene at MED, but at larger distance they fail to dope graphene. In this respect, the experiments for $\mathrm{Ni}-\mathrm{G}$ and $\mathrm{Cu}-$ G contacts report a large $R_{C}$ (especially at $V_{B G}=0$ ), thus suggesting $d>0.5 \mathrm{~nm}$. This increased distance may be the result of wrinkles, roughness and/or impurities impacting the quality of the $\mathrm{M}-\mathrm{G}$ interface.

For the $\mathrm{Au}-\mathrm{G}$ contact, a longer range interaction and significant $\mathrm{G}$ doping are predicted for $d$ up to $1 \mathrm{~nm}$, explaining the lower experimental $R_{C}$ compared to $\mathrm{Ni}-\mathrm{G}$ and $\mathrm{Cu}-\mathrm{G}$. Furthermore, the simulations predict a large $R_{C}$ dependence on $V_{B G}$ at $d>0.4 \mathrm{~nm}$. This result seems to grasp the experimental behavior, thus supporting the picture of a $\mathrm{M}-\mathrm{G}$ distance larger than the minimum distance.

\section{ACKNOWLEDGEMENTS}

Work was funded by the EU through the FP7 STREP Project GRADE (317839) and an ERC grant (InteGraDe, 307311). The German Research Foundation is acknowledged.

\section{REFERENCES}

[1] Y. Wu, X. Zou, M. Sun, Z. Cao, X. Wang, S. Huo, J. Zhou, Y. Yang, X. Yu, Y. Kong, G. Yu, L. Liao, and T. Chen, "'200 GHz maximum oscillation frequency in CVD graphene radio frequency transistors,", ACS Applied Materials and Interfaces, vol. 8, pp. 25 645-25 649, 2016.

[2] C. Chen and J. Hone, "Graphene nanoelectromechanical systems," Proceedings of the IEEE, vol. 101, no. 7, pp. 1766-1779, 2013.

[3] S. Venica, F. Driussi, P. Palestri, D. Esseni, S. Vaziri, and L. Selmi, "On the adequacy of the transmission line model to describe the graphenemetal contact resistance," IEEE Trans. on Electron Devices, vol. 61, no. 7, pp. 2570-2576, 2014.

[4] A. Meersha, H. B. Variar, K. Bhardwaj, A. Mishra, S. Raghavan, N. Bhat, and M. Shrivastava, "Record low metal - (CVD) graphene contact resistance using atomic orbital overlap engineering," in IEEE IEDM Technical Digest, 2016, p. 5.3.15.3.4.

[5] S. Venica, F. Driussi, A. Gahoi, S. Kataria, P. Palestri, M. C. Lemme, and L. Selmi, "Reliability analysis of the metal-graphene contact resistance extracted by the transfer length method," Proceedings of ICMTS, pp. 57-62, 2018.

[6] A. Gahoi, S. Wagner, A. Bablich, S. Kataria, V. Passi, and M. C. Lemme, "Contact resistance study of various metal electrodes with CVD graphene," Solid State Electronics, vol. 125, pp. 234-239, 2016.

[7] S. Venica, F. Driussi, A. Gahoi, V. Passi, P. Palestri, M. C. Lemme, and L. Selmi, "Detailed characterization and critical discussion of series resistance in graphene-metal contacts," Proceedings of ICMTS, pp. 27$31,2017$.

[8] F. Driussi, S. Venica, A. Gahoi, A. Gambi, P. Giannozzi, S. Kataria, M. C. Lemme, P. Palestri, and D. Esseni, "Improved understanding of metal-graphene contacts," Microelectronic Engineering, vol. 216, p. 111035, 2019.

[9] S. Venica, F. Driussi, A. Gahoi, P. Palestri, M. C. Lemme, and L. Selmi, "On the adequacy of the transmission line model to describe the graphene-metal contact resistance," IEEE Trans. on Electron Devices, vol. 65, no. 4, pp. 1589-1596, 2018.

[10] P. A. Khomyakov, G. Giovannetti, P. C. Rusu, G. Brocks, J. van den Brink, , and P. J. Kelly, "First-principles study of the interaction and charge transfer between graphene and metals," Physical Review B, vol. 79, p. 195425, 2009.

[11] P. Giannozzi, O. Andreussi, T. Brumme, O. Bunau, M. B. Nardelli, M. Calandra, R. Car, C. Cavazzoni, D. Ceresoli, and M. Cococcioni, "Advanced capabilities for materials modelling with quantum espresso," Journal of Physics: Condensed Matter, vol. 29, no. 46, p. 465901, 2017.

[12] C. Gong, G. Lee, B. Shan, E. M. Vogel, R. M. Wallace, , and K. Cho, "First-principles study of metalgraphene interfaces," Journal of Applied Physics, vol. 108, no. 12, p. 123711, 2010.

[13] J. Gebhardt, F. Viñes, and A. Görling, "Influence of the surface dipole layer and Pauli repulsion on band energies and doping in graphene adsorbed on metal surfaces," Phys. Rev. B, vol. 86, p. 195431, 2012.

[14] G. Henkelman, A. Arnaldsson, and H. Jonsson, "A fast and robust algorithm for bader decomposition of charge density," Computational Materials Science, vol. 36, pp. 354-360, 2006. 